\title{
UCRL-TR-208720
}

LAW RENCE LIVERMORE N A TIO N A L LABORATORY

X-Ray Raman Spectroscopy of High-Pressure Phases and Novel Materials derived from Low- $Z$ Compounds

W. J. Evans, P. J. Eng, B. Maddox, M. Newville, B. Baer, H. Cynn, M. J. Lipp, V. lota-Herbei, C.-S. Yoo

December 20, 2004 
This document was prepared as an account of work sponsored by an agency of the United States Government. Neither the United States Government nor the University of California nor any of their employees, makes any warranty, express or implied, or assumes any legal liability or responsibility for the accuracy, completeness, or usefulness of any information, apparatus, product, or process disclosed, or represents that its use would not infringe privately owned rights. Reference herein to any specific commercial product, process, or service by trade name, trademark, manufacturer, or otherwise, does not necessarily constitute or imply its endorsement, recommendation, or favoring by the United States Government or the University of California. The views and opinions of authors expressed herein do not necessarily state or reflect those of the United States Government or the University of California, and shall not be used for advertising or product endorsement purposes.

This work was performed under the auspices of the U.S. Department of Energy by University of California, Lawrence Livermore National Laboratory under Contract W-7405-Eng-48. 


\title{
Advanced Photon Source Activity Report 2003: Reports of Work Conducted at the APS, January 2003-December 2003
}

\section{X-Ray Raman Spectroscopy of High-Pressure Phases and Novel Materials derived from Low-Z Compounds}

\author{
W.J. Evans, ${ }^{1}$ P.J. Eng, ${ }^{2,3}$ B. Maddox, ${ }^{1}$ M. Newville, ${ }^{3}$ B. Baer, ${ }^{1}$ H. Cynn,,${ }^{1}$ M.J. Lipp, ${ }^{1}$ V. Iota-Herbei ${ }^{1}$ and C.-S Yoo ${ }^{1}$ \\ ${ }^{1}$ High Pressure Physics Group, H-Division, Physics \& Advanced Technologies, Lawrence Livermore National Laboratory, \\ Livermore, CA U.S.A.; ${ }^{2}$ GSECARS, Consortium for Advanced Radiation Sources, The University of Chicago, Chicago, IL, \\ U.S.A.; ${ }^{3}$ James Franck Institute, The University of Chicago, Chicago, IL U.S.A.
}

\section{Introduction}

We have used X-ray Raman spectroscopy (XRS) to study the oxygen and carbon $\mathrm{K}$-edges of high pressure phases of $\mathrm{CO}$ and $\mathrm{CO}_{2}$ to understand changes in chemical bonding associated with the pressure-induced molecular-to-nonmolecular phase transitions in these materials. This work has exploit the power of the XRS technique to resolve important scientific questions regarding the nature of the high-pressure phases of $\mathrm{CO}$ and $\mathrm{CO}_{2}$.

The application of high pressures often transforms molecular solids to nonmolecular extended solids, manifesting entirely different chemical bondings and crystal and electronic band structures. Because of a sizeable difference in bondings and a large activational energy, these novel forms of high-pressure phases are often metastably recoverable, yet inaccessible to conventional chemical synthesis techniques at ambient pressures. These new phases are often of geophysical interest because the pressure/temperature-synthesis conditions are relevant to planetary interiors and in many cases the crystal structures are commonly shared by terrestrial minerals. The classic example of such a system is diamond, and recent work, including research of the proposing group, has found new examples including novel phases derived from Carbon Monoxide [1] and Carbon Dioxide [2]. Kinetic barriers at high pressures often hinder the transformations leading to incomplete or disordered products. Thus x-ray diffraction may not definitively identify the new phase and additional diagnostic studies can serve to validate phase and structural identifications. Recent X-ray Raman studies of the oxygen K edge by Bergmann et al. at APS Sector 18 demonstrate its power in studying the local structure of water molecules [3].

At pressures of $\sim 50 \mathrm{kbar}$, the $\mathrm{CO}$ molecular solid transforms to a novel phase that loses it's molecular signature and, we contend, interconnects to form a covalently bonded network (extended solid) [1]. The high-pressure phase of CO can be quenched and is metastable at ambient pressure and temperature. The ambient condition metastability/lifetime of the CO-derived solid can vary from minutes to seemingly indefinite.

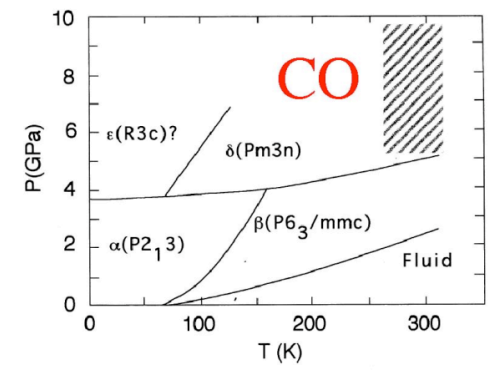

Fig. 1. Phase diagram of $C O$, adapted from reference 1. We hypothesize that this variation in lifetime is related to the degree of polymerization. We have performed various optical spectroscopies on this material, but a definitive conclusion remains elusive and the absorption band of the diamond anvil obscures important regions of the infrared spectrum. The recovered materials also appear to be amorphous, so x-ray diffraction provides only limited information. Using XRS we will study the oxygen and carbon K-edges to observe timedependent changes at high pressure and in the quenched metastable phase. Information on the electronic environment of the oxygen and carbon atoms can yield insight into both the transformation and decomposition processes. This material is energetic and may serve as a high-density fuel for propulsion applications [4].

In our recent studies, we have discovered that molecular $\mathrm{CO}_{2}$ (cubic phase I) transforms to a covalently bonded extended solid (tridymite-like phase $\mathrm{V}$ with four-fold coordinated carbon) above $40 \mathrm{GPa}$ and $2000 \mathrm{~K}$ [2]. This transformation is believed to occur as a result of pressure-induced electron delocalization. However, the exact mechanism is not very well understood as yet. For example, the presence of intermediate phases such as pseudo-six-fold $\mathrm{CO}_{2}$-phase II and bent $\mathrm{CO}_{2}$-phase IV suggests that the electron delocalization apparently occurs continuously with increasing pressures above 10-20 GPa $[5,6]$.

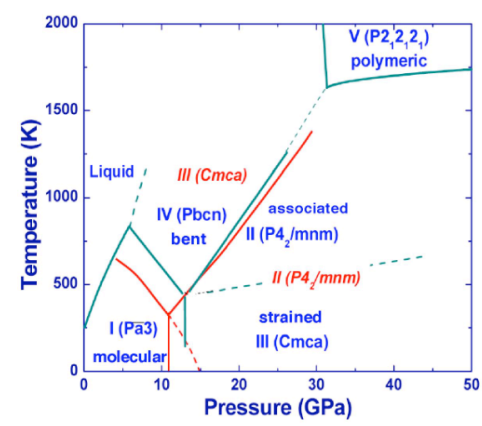

Fig. 2. Phase diagram of $\mathrm{CO}_{2}$. Blue lines established by experiment, red lines indicate theoretical predictions. Adapted from ref.s 6 and 7.

A recent theoretical work, on the other hand, suggests a quite different "abrupt" mechanism [7]. One way to clarify this discrepancy is to directly determine the change in chemical bonding in carbon dioxide at high pressures, which should be reflected in the near-K-edge absorption spectra of oxygen and carbon. 
Both carbon and oxygen have very low energy x-ray absorption bands, $\sim 290 \mathrm{eV}$ and $\sim 540 \mathrm{eV}$ for the carbon and oxygen Kedges respectively, which present a severe challenge for $\mathrm{x}$-ray absorption spectroscopy. The x-rays at this energy cannot penetrate through the relatively thick and absorptive samples and diamond anvils. However, this limitation can be overcome using the XRS technique of measuring inelastically scattered high-energy x-rays. The performance characteristics of a highintensity third-generation synchrotron source like the APS are ideally suited and crucial to this experiment. Several sectors at the APS, including Sectors 18 and 13, have demonstrated such experiments in several low-Z materials like $\mathrm{H}_{2} \mathrm{O}$ [3] and carbon [8] at both ambient and high pressures.

\section{Methods and Materials}

In this study, $\mathrm{CO}_{2}$ and $\mathrm{CO}$ samples were be loaded cryogenically in diamond anvil cells with beryllium gaskets ( $\mathrm{x}$-ray transparent) and a few ruby particles for sensing the pressure. Typical sample sizes were $30 \mu \mathrm{m} \times 100 \mu \mathrm{m}$ (thickness, diameter). Diamond anvil cells manufactured with a large conical aperture perpendicular to the load axis were used. The x-ray beam $(\sim 100$ $\mu \mathrm{m}$ spot size) was incident upon the sample through the a small hole in the DAC and then the beryllium gasket. The scattered $\mathrm{x}$ rays were collected at $\sim 30$ degrees from the incident beam. The conical aperture permited collection of a large solid angle of $\mathrm{x}$ rays scattering and exiting through the gasket. The incident high-energy monochromatic x-ray was at an energy of $\sim 10 \mathrm{keV}$. Following the work of previous researchers $[3,9]$, we will scan the incident $\mathrm{x}$-ray monochromator over a range of 50-100 eV. The inelastically scattered photons were detected using a sixcrystal analyzer (resoln. $1.7 \mathrm{eV}$ ) at a fixed energy, $\mathrm{E}_{\mathrm{f}}$, using a multi-crystal analyzer and a liquid-nitrogen-cooled $\mathrm{Ge}$ detector. Thus the Raman shift $\mathrm{DE}=\mathrm{E}_{0}-\mathrm{E}_{\mathrm{f}}$ is determined by scanning $\mathrm{E}_{0}$, rather than the conventional optical approach of a fixed $\mathrm{E}_{0}$ and a scanned $E_{f}$. Typical times for collection of XRS spectra were 6-8 hours.

\section{Results}

Oxygen and Carbon K-edge XRS spectra were successfully collected for $\mathrm{CO} 2$. Unfortunately the $10 \mathrm{keV} x$-ray induced radiation damage and/or the transformation of $\mathrm{CO}$ to an unidentified phase, possibly related to a polymeric phase of $\mathrm{CO}$ that has been produced at high pressure [4]. Based on an independent set of experiments, we believe this transformation occurs quite rapidly, on timescales of minutes to hours. Because of the long collection times the XRS spectra of the $\mathrm{CO}$ is certainly a mixture of pure $\mathrm{CO}$ and transformed material with unknown relative strengths.

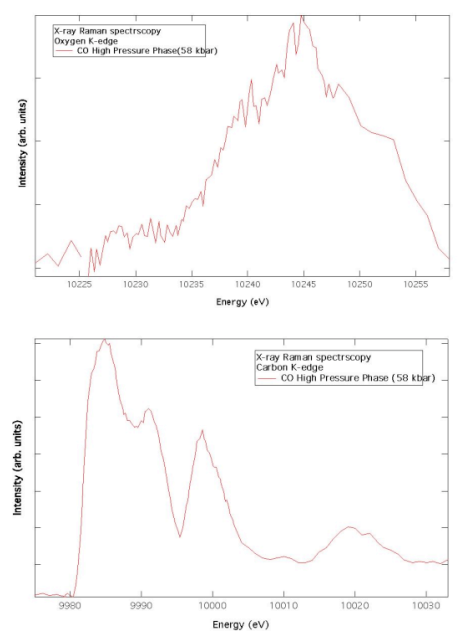

Fig. 3. Oxygen and carbon k-edge XRS spectra of $\mathrm{CO}$ at 58 kbar. Radiation induced damage/transformation altered the nature of the sample during data collection

In contrast we met with more success ion studies of $\mathrm{CO}_{2}$. Below we show XRS spectra of different phases of $\mathrm{CO}_{2}$.
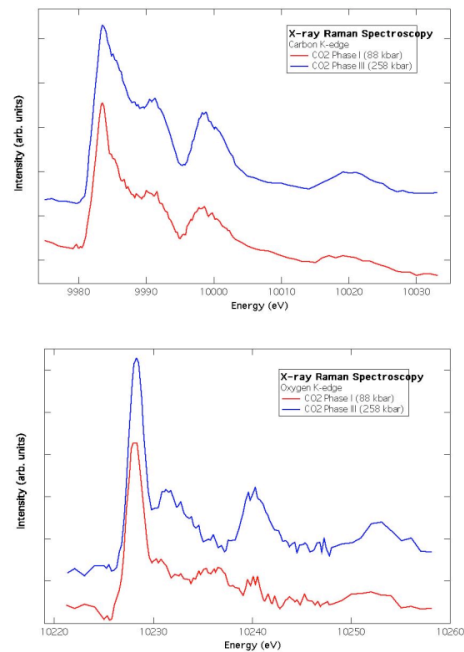

Fig. 3. Oxygen and carbon k-edge XRS spectra of $\mathrm{CO}_{2}$ at $58 \mathrm{kbar}$.

Carbon k-edge XRS spectra are to be treated with some reservations since the carbon in the diamond anvil cannot be eliminated and may enter into the measured data.

\section{Discussion}

The XRS oxygen k-edge spectra are the most readily tractable data from our studies. These data do not have any radiation damage/conversion or diamond anvil contaminations issues.

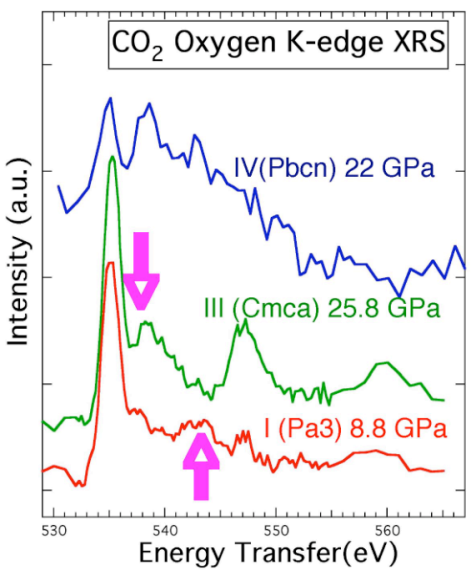

Fig. 3. Oxygen k-edge XRS spectra of $\mathrm{CO} 2$ in various highpressure phases. New peaks and changes are noted by arrows.

Initial review of the XRS data identify clear differences in the spectra in the experimentally identified phases, supporting the contention of changes in not only crystal structure but chemical bonding in phases II, III, and IV (associated molecular vs molecular). Clear differences are observed in the spectra, and detailed analysis should yield information on the local structure of these phases. Continued study and analysis is underway including

- improved studies (larger samples, higher $\mathrm{S} / \mathrm{N}$ ) of $\mathrm{CO}_{2}$ (II, IV \&V), particularly the polymeric phase V. 
- develop a more quantitative and theory-based interpretation of XRS spectra in relation to electronic changes in high-pressure phases

- $\quad$ study pressure dependence of XRS within phases as compared to across phase lines.

- $\quad$ apply XRS to other molecular systems of interest.

\section{Acknowledgements}

This work was performed under the auspices of the U.S. DOE by the Univ. of Calif., LLNL under contract No. W-7405-Eng48 and at GeoSoilEnviroCARS, by the NSF- Earth Sciences (EAR-0217473), DOE - Geosciences (DE-FG02-94ER14466) and the State of Ill. Use of the Advanced Photon Source was supported by the U.S. Department of Energy, Office of Science, Office of Basic Energy Sciences, under Contract No. W-31-109-ENG-38.

\section{References}

[1] M. Lipp et al., Journal of Low Temperature Physics, 111(3/4), 247 (1998).

[2] V. Iota et al., Science 283, 1510 (1998).

[3] U. Bergmann et al., Physical Review B, 66, 092107 (2002).

[4] M. Lipp et al., Nature Materials, accepted for publication, December 2004.

[5] C.-S. Yoo et al., Physical Review B, 65, 104145 (2002).

[6] V. Iota and C.-S. Yoo, Physical Review Letters, 86, 5922

(2001).

[7] S. Bonev et al., Physical Review Letters, 93, 65501-1

(2003).

[8] W. Mao et al., Science, 302, 425 (2003).

[9] U. Bergmann, P Glatzel and S.P. Cramer, Microchemical Journal, 71(2-3), 3121 (2002). 